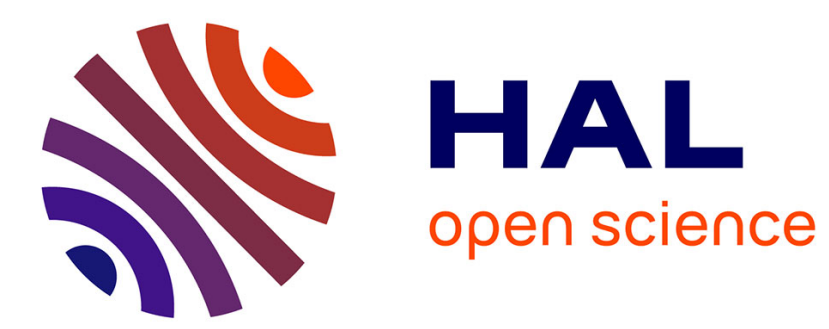

\title{
INTERPRETATION OF MULTIPLICITY DISTRIBUTIONS
}

\author{
Eric Berger
}

\section{To cite this version:}

Eric Berger. INTERPRETATION OF MULTIPLICITY DISTRIBUTIONS. Journal de Physique Colloques, 1973, 34 (C1), pp.C1-346-C1-351. 10.1051/jphyscol:1973145 . jpa-00215225

\section{HAL Id: jpa-00215225 https://hal.science/jpa-00215225}

Submitted on 1 Jan 1973

HAL is a multi-disciplinary open access archive for the deposit and dissemination of scientific research documents, whether they are published or not. The documents may come from teaching and research institutions in France or abroad, or from public or private research centers.
L'archive ouverte pluridisciplinaire HAL, est destinée au dépôt et à la diffusion de documents scientifiques de niveau recherche, publiés ou non, émanant des établissements d'enseignement et de recherche français ou étrangers, des laboratoires publics ou privés. 


\section{INTERPRETATION OF MULTIPLICITY DISTRIBUTIONS}

\section{E.L. BERGER}

CERN and Argonne National Laboratory

1.- Data on mitiplicity distributions in protion proton interactions at high-energies, which first become available a year or so ago, provide valuable qualitative insight into some global aspects of hadron production [1] . Further bubble chamber investigations at Serpukhov and at NAL during the past year $[2,3]$ have added more detail on the energy (s) and multiplicity $(n)$ dependence of the partial crosssections $\sigma_{n}(s)$. At the same time, single- and two-particle inclusive momentum distributions have been obtained over the energy range 30 to $1500 \mathrm{GeV} / \mathrm{c}$. Important analyses of the properties of selected exclusive channels are also beginning. It seems clear that proper interpretation of multiplicity spectra requires simultaneous consideration of all this other relevant information. A clear trend in this direction is visible in papers written over the past year. In some of the more interesting recent work, a serious attempt is made to integrate knowledge of multiplicity distributions, momentum spectra, and various correlations.

My charge is to report on multiplicities as such, and to leave the treatment of detailed models and of momentum spectra to others. I will concentrate primarily on pp results, since they are the most complete. In large measure, the pp data are also representative of the general picture, although interesting systematic differences are present between particle-antiparticle processes [3] (e.g. $K^{ \pm} p$ at $32 \mathrm{GeV} / \mathrm{c}$ ).

2. - EARLY LESSONS. - Several important observations based on the early data have been confirmed by more recent measurements. The slow growth with $s$ of the mean number of charged particles may be parametrized as $\left\langle n_{c h}\right\rangle \simeq a+b \log s$ or as $\left\langle n_{c h}\right\rangle \sim s^{1 / 3}$, (with additional "threshold" correction terms in both expansions). Coefficient $b$ has values ranging between 1.4 and 2.0 depending upon whether threshold terms are used $[4,5]$. The mean spacing $\langle\Delta y\rangle$ between hadrons in rapidity space is therefore small :
$\left\langle\Delta_{y}\right\rangle \simeq \underline{\simeq} 0.3-0.5$. In terms of mean invariant mass of hadron pairs, this implies $\left\langle\Delta_{\mathrm{m}}\right\rangle \simeq 400-500 \mathrm{MeV}$. obviously, independent emission of hadrons is excluded, and one must expect important final-state interaction effects, clustering, correlations and so forth. Second, the two-prong $\left(\sigma_{2}\right)$ and four-prong $\left(\sigma_{4}\right)$ pp cross-sections are observed to fall continuously with $s$ from 30 to $400 \mathrm{GeV} / \mathrm{c}$. A crude parametrization [4] gives $\sigma \sim \mathrm{p}_{\mathrm{Lab}}^{-0.4}$. It is therefore by no means clear from these data that an energy-independent ("diffractive") non-zero lower bound is being approached. In pp collisions, perhaps the best way to establish the existence and magnitude of such a lower limit will be to study systematically the $s$ dependence of selected exclusive channels (e.g. pp $\rightarrow$ pp $\pi^{+} \pi^{-}$). Third, the full $n$ dependence of $\sigma_{n}$ at large $s$ is described well neither by a Poisson formula nor by $\sigma_{n} \propto n^{-2}$. Nevertheless, it is clear that a poisson form is a much better representation of the large $n(n\rangle\langle n\rangle)$ behaviour than is $\sigma_{n} \sim n^{-2}$. Simple diffractive models [6] for high $n$ are excluded. Fourth, correlation parameter $f_{2}=\langle n(n-1)\rangle-\langle n\rangle^{2}$ grows increasingly positive as $s$ increases. Fluctuations about the mean are significant. The $s$ dependence of $f_{2}$ is in principle a good discriminator between models. In practice, however, data can be fitted with expressions whose leading $s$ behaviour is logs, $(\operatorname{logs})^{2}, \sqrt{s}$, or $s$, and so we must look deeper. Fifth, a simple rule appears to describe the growth of the width $D=\left(\left\langle n^{2}\right\rangle-\langle n\rangle^{2}\right)^{1 / 2}$. Data can be fitted as

$$
D=(0.58 \pm 0.01)\left[\left\langle n_{c h}\right\rangle-1\right] .
$$

This regularity was first suggested by Malhotra [7] in 1963 and later explored in more detail by Czyzewski and Rybicki (C.R) [8] and by Wroblewski [9].

\section{3. - MORE RECENT EFFORTS. - Investigations of the} past year may be placed in five categories. First, 
In a number of papers, the authors seek to fit $\sigma_{n}(s)$ with one smooth function of $n$ and $s$, with few parameters, Basically, these are modifications of probability distributions [10] $\mathrm{P}_{\mathrm{n}}=\sigma_{\mathrm{n}} / \sigma$. Little or no attempt is made to justify physically or to derive these functions from models. In the second and third categories I place the Koba-Nielsen-olesen (KNO) scaling [11] and "two-component" approaches. Fourth, interesting information is available on associated multiplicities, neutral-charged correlations, left-right hemisphere multiplicity correlations, and the like. In the final category, I collect fits to (or implications for) $\sigma_{n}(s)$ obtained from detailed dynamical models $[12,13]$, in which an attempt is made to accommodate simultaneously data on $\sigma_{n}(s)$, momentum spectra and correlations.

Annong papers dealing with one smooth function for $\sigma_{n}(s)$, I may mention (a) the C-R formula, now extended to neutral-charged correlations [14], (b) approximately normal distributions [15], and (c), the Buras-Koba-Weisberg formula [16]. A.11 of these are advertized as extremely good fits, and, in most cases, they are good by any $\chi^{2}$ test. However, a relevant question is raised by Kaiser [15], among others. Given the considerable structure seen for example in the inclusive proton spectrum, what right do we have to expect a smooth function $\sigma_{n}(s)$ ? If the final $\sigma_{n}(s)$ really is featureless, we have to explain why it should be so, in contrast to single particle momentum spectra. One speculation, investigated by the ABCLV collaboration [17], is that the charged - multiplicity distribution is smoothed out, whereas the true-multiplicity distribution has considerable structure even at relatively low energy. I find that there is little justification for supposing that one smooth function should fit, and essentially no predictive value in the results.

4. - KNO SCALING. - This heading covers one of the most hotly debated subjects of the past year. The scaling rule states that

$$
P_{n}(s)=\frac{\sigma_{n}(s)}{\sigma(s)}=\frac{1}{\langle n\rangle} \psi\left(\frac{n}{\langle n\rangle}\right) \text {. }
$$

Its original "derivation", based on the assumption of Feynman scaling for all inclusive spectra [11], can be meaningful and valid only at extraordinarily high $s$, if at all. One begins with the identity

$\sigma\langle n(n-1) \ldots(n-q+1)\rangle=\int \ldots \int d_{1} \ldots d_{q} \frac{d^{y_{\sigma}}}{d y_{1} \ldots d y_{q}} .(3)$
If the q-particle inclusive cross-section has attained an energy independent plateau, the integral gives $C_{q}(\log s)^{q}+o\left[(\log s)^{q-1}\right]$, where $c_{q}$ is a constant. Therefore, we deduce

$$
R_{q}=\frac{\left\langle n^{q}\right\rangle}{\langle n\rangle^{q}}=c_{q}\left\{1+o\left(\frac{1}{\langle n\rangle}\right)\right\}
$$

Correction terms of order $\langle n\rangle^{-1}$ are indicated. The ratio $R_{q}$ can be expected to be represented by the right hand side of equation 4 only for $q\langle\langle n\rangle$. If equation 4 holds for all $q$, then (4) implies (2).

Because an approximately smindependent plateau even for single particle spectra is not observed to set in until ISR energies, if at all, the derivation shows that only $q \leq 2$ satisfies the basic postulate. Analyses of multiplicity data [18] show, however, that $R_{q}$ is roughly constant for $p_{l a b} \geq 50 \mathrm{GeV} / \mathrm{c}$ and $q \leq 5$. Further, a universal function $\$(z=n /\langle n\rangle)$ as defined in equation (2) seems to fit pp data in the range $50 \leq \mathrm{p}_{1 a b} \leq 300 \mathrm{GeV} / \mathrm{c}$. An example [19] of such a fit is given in Fig. 1.

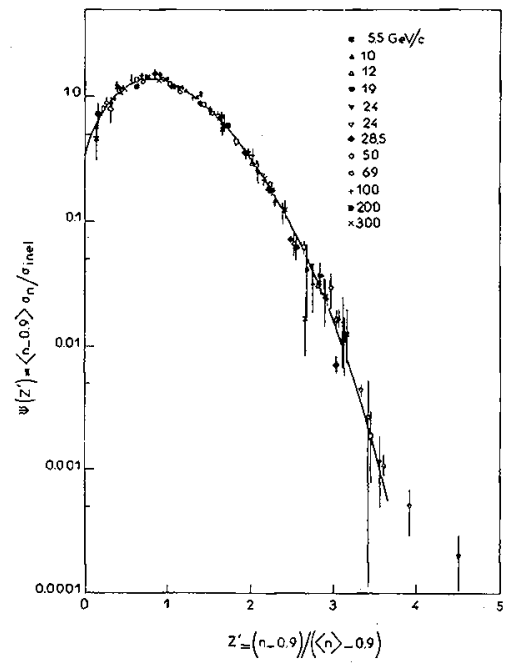

Fig.1 - plot of a modified KNO scaling function $\psi\left(z^{\prime}\right)$ vs. $z^{\prime}$. Multiplicity data from pp interactions are shown for laboratory momenta ranging from 5.5 to $300 \mathrm{GeV} / \mathrm{c}$. Variable $z^{\prime}=(n-0.9) /(\langle n\rangle-0.9)$. This figure is taken from Ref.19, in which references to data may also be found.

In view of the fact that the required s-independent plateaux are not observed, this early onset of apparent kNO scaling clearly has absolutely nothing to do with the original derivation. Is the precocious appearance of a universal function $\psi(z)$ profound or accidental ? If accidental or transitory, we may expect that as $s$ increases further, the function $\Psi(z)$ will actually change gradually before approaching 
its true scaling limit $\Psi_{\infty}(z)$, if such a $\psi_{\infty}$ exists. Supporters of the idea that the early scaling is profound search for models which incorporate this feature.

The accidental/profound debate is not resolved. Data on the large $n$ behaviour of $\sigma_{n}$ vs $n$ in the energy range $100 \leq p_{1 a b} \leq 400 \mathrm{GeV} / \mathrm{c}$ are of $11-$ mited statistical significance, so that deviations from a universal fit are easily hidden. Perhaps a more sensitive test of rellability is provided by the $s$ dependence of the ratio $[\operatorname{mode} /\langle n\rangle]$. As remarked by Wroblewski [9], data on this quanti ty show a systematic decrease with $\mathrm{p}_{\text {lab }}$, whereas a constant ratio is demanded if early kNO is true.

second, a purely heuristic derivation of early kNO scaling may be given. The distribution $\mathbf{P}_{\mathbf{n}}=\sigma_{\mathrm{n}} / \sigma$ plotted versus $n$ has unit area at all $s$, but its shape spreads to larger $n$ as $s$ increases. If the $x$-axis is scaled by $\langle n\rangle$ and the $y$ axis multiplied by $\langle\pi\rangle$, unit area is preserved. However, the distributions at different $s$ collapse approximately to one universal distribution provided that the ratio $D /\langle n\rangle$ is constant. (D is the "width" of the distribution, Eq.(1)). This observation reduces the apparent early onset of KNO simply to a manifestation of the Malhotra-Wroblewski rule, at least to first order. To be sure, true early KNO scaling requires that the next moment, skewness $\gamma_{3}$, also be s-independent

$$
\gamma_{3}=\frac{\left\langle(n-\langle n\rangle)^{3}\right)}{\langle n\rangle^{3}} \text {. }
$$

However, this quantity is relatively small.

Third, why should KNO scaling work when applied to charged multiplicity $\mathrm{n}_{\mathrm{ch}}$ and not to negative multiplicity $n_{-}$? For negatives $\gamma_{2}$ and $\gamma_{3}$ fall systematically [12] with s.

Fourth, many approaches, including the "twocomponent" approach fit $\sigma_{n}$ data perfectly over the energy range 50 to $300 \mathrm{GeV} / \mathrm{c}$. In this sense, these models provide a function $\psi(z)$ definable from their "perfect" fits in this energy range $[20,21]$. However, for most of these approaches the asymptotic function $\psi_{\infty}(z)$ is far from that deduced from present data. These fits suggest therefore that apparent KNO scaling in the present enerfy range is entirely transitory. The scale of energies_[21] over which things enange may be of order $10^{4} \mathrm{GeV}$. Demonstrations of this sort may be as much against the specific models as they are against early kNO scaling, of course. However, their existence dampens enthusiasm for the empirical regularity, and surely undermines credence in extentions of KNO fits to $\pi^{\circ}$ and $K$ multiplicity distributions for which statistics are even less impressive [22].

Absence of a solid derivation or of strong theoretical motivation, coupled with phenomenological doubts of the type mentioned above, suggest that the real kNO scaling limit, if any, may be very different from that suggested by present analyses. Nevertheless, there has been very interesting spinoff from the efforts of the pro-KNO school. I may call attention especially to the work of Koba and of Koba and Weingarten on density correlations and normalized cumulants [23]. Models in which KNO scaling is built in from the start include several impact-parameter models, which are quite interesting in their own right $[24,25]$.

5. - MULTI-COMPONENT APPROACH, - The physical motivation for this general view point seems especialIy strong. Observations of inclusive momentum distributions and of exclusive channels give tentative evidence for contributions from different reaction mechanisms : single inelastic diffraction both of low mass and of high mass states, "central" production of pions and of anti-baryons, non-diffractive peripheral mechanisms, and so forth. Each of these mechanisms may have a characteristic and different $\sigma_{n}(s)$. We observe the sum of all effe ts. It is an intriguing experimental challenge to find operational methods for separating various mechanisms.

Although the physical motivation is high, twocomponent fits to $\sigma_{n}(s)$ involve great freedom and many arbitrary assumptions. In practice, the two-component approach has meant the addition of contributions from two overly simple components[26, 27] . An approximately Poisson distribution is fitted to the high multiplicity tail, and then enough is added to the $\sigma_{n}$ at low multiplicities to produce a perfect fit. The low-multiplicity add-on is often loosely termed "diffractive". For the high-n component, each $\sigma_{n} \rightarrow 0$ asymptotically, and $\left\langle n_{h i g h}\right\rangle \sim$ b logiswith $b_{c h} \sim 2$ to 3 . Even less is generally true about the low-n component ; crudely, $\left\langle\mathrm{n}_{10 w}\right\rangle$ grows slowly with $s$, if at all, and either each $\sigma_{n D} \rightarrow \mathbf{C}_{\mathbf{n}} \neq 0$ or $\sum_{n} \sigma_{n D}=$ constant.

Several further remarks may be made. First, from the $\sigma_{n}(s)$ data alone, one cannot prove the need for more than one (pure-short-range correlation) component. An acceptable fit to data with $\mathrm{p}_{1 \mathrm{ab}} \geq$ $50 \mathrm{GeV} / \mathrm{c}$ may be constructed in which one defines $\left\langle n_{-}\right\rangle, f_{2}^{(-)}$to agree with data [26]. Both have leading $s$ dependence given by logs ; curvature of 
$\left\langle n_{-}\right\rangle$and $f_{2}^{(-)}$is accommodated by non-asymptotic terms falling as $(\sqrt{s})^{-1}$. Second, if the model is defined to be one in which there are two simple components, one invariably finds $\sigma_{\text {low }} / \sigma \simeq 0.2$. This result is obtained upon forcing agreement with the Malhotra-Wroblenski regularity [28]. Third, the present two-component approach leads to an asymptotic prediction $r_{3}<0$. This is qualitatively obvious inasmuch as the approach provides a low multiplicity shoulder, of smaller overall magnitude than the dominant high multiplicity component. Thus, skewness eventually becomes negative. Proponents of early kNO scaling view this feature as a possible fatal flaw of the two-component view-point [29]. Data on $f_{3}^{\text {ch }}$ are roughly energy independent over the present energy interval. Fourth, a dip structure, the "wilson-dip", may appear at high enough energies if the low and high $n$ components separate. Its observation would be impressive support for twoor multi-component pictures, and would help nail down parameters. However, no dip in the ISR energy range can really be said to be expected from fits to lower energy data, in the sense that present parameters are too poorly determined.

Finally, any number of paralyzing objections may be made against present fits. Poisson forms for the high-n component are of doubtful justification. Correlations, clustering, and energy-momentum effects surely break any Poisson assumption. Further, obvious efforts are needed to justify assertions about the low $n$ component. The notion of diffraction is one based on observation of final proton momentum distributions. Thus, experimental selections to isolate operationally a diffractive subsample should provide a less ambiguous definition of a low multiplicity component $[30]^{\dagger}$. Likewise, it would be very instructive to examine the structure of $\sigma_{n}$ vs. $n$ for events in which other possible mechanisms play a role (e.g. events with a $\pi$ in the central region, a $\bar{B}$, a large $p_{T}$ hadron, etc.). It is crucial to break-away from the idea that one must be totally inclusive. Appropriate cuts (semi-inclusive) may be very instructive.

On the theoretical front, efforts are under way to develop multi-component models with more justification and/or predictive power $[12,31]$. Triple-Regge expressions provide parametrizations of missing mass $M$ and $t$ dependence in reactions of the form $p p \rightarrow p X$. Assumptions may then be made about the dependence of multiplicity on missing mass, for each Regge exchange. Comparisons are possible. with data in selected regions of $t$ and $M^{2}$, as well as for the full distribution $\sigma_{n}(s)$. An example of results obtained from this type of approach is given in fígure 2 .

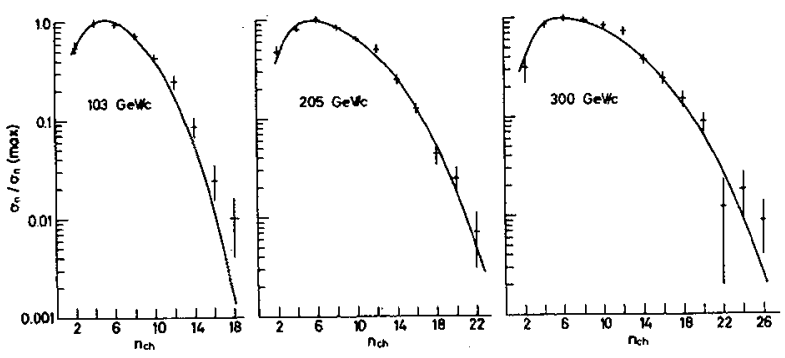

Fig.2 - The distribution $\mathrm{P}_{n}=\sigma_{n} / \sigma_{n}(\max )$ is plotted versus $n$. Data (Ref. 1 ) are shown at three energies. Theoretical curves are computed from a multiperipheral two-component model, Ref.12, which also successfully describes data on single-particle and two-particle inclusive momentum spectra in the NAL and ISR energy ranges.

6. - ASSOCIATED-MULTIPLICITIES. - One example of associated multiplicity was described in the previous section. The mean charged multiplicity $n\left(M_{x}\right)$ has been obtained from NAL data on $\mathrm{pp} \rightarrow \mathrm{pX}$ as a function of missing mass $[30,32]$. A compilation is given in figure 3.

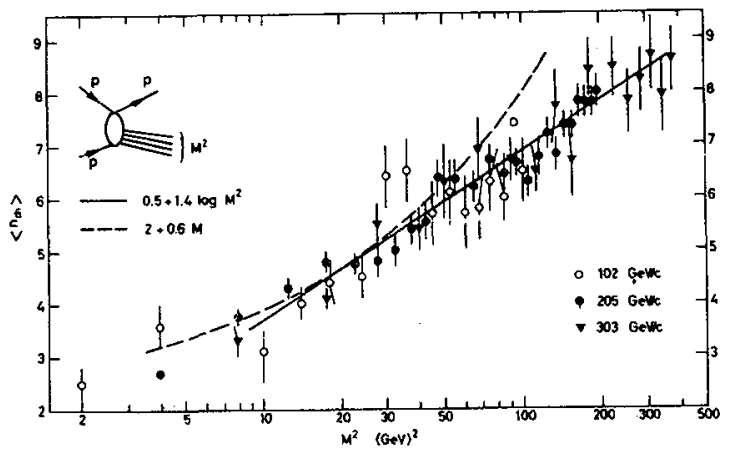

Fig. 3 - The total mean charged multiplicity observed in the reaction $\mathrm{pp} \rightarrow \mathrm{pX}$ is plotted as a function of mass squared of system $X$. Data are taken from Refs. 30 and 32. Two curves are drawn for comparison. Neither is a "best-fit".

Observe that $n\left(M_{x}^{2}\right)$ appears not to depend on energy $s$. It grows slowly with $\mathrm{M}_{\mathrm{x}}^{2}$. Two curves are drawn on figure 3 to provide a basis for comparison. Neither curve is in any sense a best fit. I took the coefficient $b=1.4$ in the parametrization

$$
\left\langle n\left(M^{2}\right)\right\rangle=a_{M}+b \log M^{2} \text {, }
$$

\footnotetext{
$\dagger_{\text {See page }} \mathbf{c 1 - 3 5 1}$
} 
directly from my fit to the $s$ dependence of overall pp charged multiplicity [4]

$$
\langle n(s)\rangle=a_{s}+b \log s \text {. }
$$

No attempt is made to reproduce threshold effects for $\mathrm{M}^{2}<10 \mathrm{GeV}^{2}$. As will be seen, the rate of $\left\langle n\left(M^{2}\right)\right\rangle$ with. $\log M^{2}$ is consistent with being the same the rate of growth of $\langle n(s)\rangle$ versus $\log s$. The linear curve

$$
\left\langle n\left(M^{2}\right)\right\rangle=0.6 \mathrm{M}+2
$$

is not an unreasonable parametrization at low $M$, but begins to fail for $\mathrm{M}^{2}>50 \mathrm{Gev}^{2}$.

Theoretical interpretation of these multiplicity data is not altogether direct. The nova approch [6] is predicated on a linear increase of multiplicity with mass of a properly defined recoil cluster. In fits to low energy data $\left[p_{1 a b} \leq 30(\mathrm{GeV} / \mathrm{c})\right]$ with this model, the form

$$
\left\langle\mathrm{n}_{\mathrm{ch}}(M)\right\rangle \simeq 1.4\left(M-\mathrm{m}_{\mathrm{p}}\right),
$$

was found appropriate (for charge multiplicity). The rather sma 11 coefficient $(0.6)$ of the linear term found in NAL data would appear to contradict nova model parametrizations, even at low $M$. This objection is not as strong as it might be, however, because the missing mass in NAL experiments is not identical to recoil cluster mass of the model. Likewise, direct interpretation of the logarithmic fit [Eq. (7)] in terms of multiperipheral-like or tripleRegge models is ambiguous inasmuch as a unique or clean exchange situation is not obtained in the data unless appropriate selections are made on $M^{2}$ and $t$.
On a purely qualitative level, the fact that the same coefficient $b \simeq 1.4$ is found in fits $\langle n(s)\rangle$ $\sim \mathrm{b} \log \mathrm{s}$ and $\left\langle\mathrm{n}\left(\mathrm{M}^{2}\right)\right\rangle \sim \mathrm{b} \log \mathrm{M}^{2}$ suggests that what is important for determining asymptotic multiplicity is the energy available (Q) in whatever system or subsystem one is dealing wish. This idea cannot be pushed too far, however, in view of the large difference observed for $f_{2}$ between $K_{p}^{ \pm}$at $32 \mathrm{GeV} / \mathrm{c}$.

$$
\begin{aligned}
& \text { 7. - CORRELATIONS. - I nasmuch as } \\
& f_{2}=\langle n(n-1)\rangle-\langle n\rangle^{2}=\iint c\left(y_{1}, y_{2}\right) d y_{1} d y_{2}
\end{aligned}
$$

measurements of $\mathrm{f}_{2}$ or of inclusive correlation function $\mathrm{C}\left(\mathrm{y}_{1}, \mathrm{y}_{2}\right)$ mix effects which arise from (a) charge-isospin correlations, (b) kinematic and dynamic momentum correlations, and (c) multiplicity correlations per se. It is desirable to separate these effects and to assess their distinct influences. It is evident that a measurement of $d^{2} \sigma / d y_{1} d y_{2}$ or of correlation function $\mathrm{C}_{n}\left(\mathrm{y}_{1}, \mathrm{y}_{2}\right)$ for fixed multiplicity $n$ would allow some separation of momentum from multiplicity correlations. Use of appropriate statistical parameters applied to individual events [33] will help to identify salient collective effects (in single events) in momentum or in rapidity space. Promising work I have done along these lines in collaboration with the Pisa-stony Brook group is deseribed by Foa in his plenary report. The measurement of correlation parameter $f_{2}^{0, O_{2}}\left(n_{-}\right)$between two neutral hadrons as a function of negative multiplicity is advertised [34] as a useful tool for identifying the isospin structure of clusters of pions emitted in a primary production mechanism.

\section{REFERENCES}

[1] Multiplicity data in pp collisions: 50 and $69 \mathrm{GeV} / \mathrm{c}$ : France-Soviet Union Mirabelle Collaboration - paper submitted to XVI Int. Conference on High Energy Physics (1972) Chicago NAL

$100 \mathrm{GeV} / \mathrm{c}$ : CHAPMAN (J.W.) et al., Phys. Rev. Letters 29 (1972) 1686

$200 \mathrm{GeV} / \mathrm{c}$ : CHARtTon (G.) et al., Phys. Rev. Letters 29 (1972) 515

$300 \mathrm{GeV} / \mathrm{c}$ : DAO (F.T.) et al., Phys. Rev. Letters 29 (1972) 1627

$400 \mathrm{GeV} / \mathrm{c}:$ Michigan Rochester Collaboration, results reported at this Conference

[2] $x^{-}$p data at $200 \mathrm{GeV} / \mathrm{c}$, Berkeley-NAL collabo-

[3] $\mathrm{K} \pm_{\mathrm{p}}$ and pp at $32 \mathrm{GeV} / \mathrm{c}, \pi^{ \pm} \mathrm{p}$ at $50 \mathrm{GeV} / \mathrm{c}$ : France-Soviet Union and CERN-Soviet Union Collaborations, contribution 413

[4] BERGRR (E.L.), Phys. Rev. Letters 29 (1972) 887

5] ANTINUCCI (M.) et al., Nuovo Cimento Letters 6
[6] BENECKE (J.) et al., Phys, Rev, 188 (1969) 2159 HWA (R.), Phys, Rev. Letters 26. (1971) 1143 JACOB (M.) and SLANSKY (R.), Thys. Rev. D5 (1972) 1847 ADAIR (R.), Phys. Rev. D5. (1972) 1105

[7] MALHOTRA (P.K.), Nuc1, Phys, 46 (1963) 559

[8] CZYZEWSKI (C.) and RYBICKI (K.), Nucl. Phys. B47 (1972) 633

[9] WROBLEWSKI (A.), Zakopane Lectures, 1973

[10] FELLER (W.), An introduction to probability theory and its application; Edit. Wiley (1958)

[11] KOBA (z.), NIELSEN (H.,.) and OLESEN (P.), Nucl. Phys. B4O (1972) 317. For a recent review, consult $z$. KOBA, Lectures presented at the 1973 CERN-JINR School, Ebeltoft

[12] BERGER (E.L.) and FOX (G.C.), CERN Report Ref. $\mathrm{TH} 1700$ to be published in Physics Letters and Conference contribution 149

[13] KRIPFGANZ ( $\left.J_{.}\right), \operatorname{RANFT}\left(\mathrm{G}_{*}\right)$, and RANFT (J.), paper 69 TER-MARTIROSYAN (K.A.), Phys, Letters 44B (1973) 377 
[14] BARDADIN-OIWINOWSKA et al. (M.), paper 379

[15] HEIKO (L.) and HVEGHOLM (J.), paper 297 KAISER (G.D.), paper 199

PARRY (G.W.) and ROTELLI (P.), Trieste preprint (1973)

[16] BURAS (A.) and rOBA (z.), Nuovo Cimento Letters 6 (1973) 629

WEISBERG (H.), Univ, of Penn. Report (1973) See also BAIER (R.) and WIDDER (F.), Graz-Wien preprints (1973)

[17] DEUTSCHMANN (M.) et al. CERN report DPh II/Phys. 73-27, paper 220

[18] SLATTERY (P.), Phys. Rev. Letters 29 (1972)1627

[19] BURAS $\left(A_{1} J_{0}\right)$, DIAS DE DEUS (J.) and MOLLER (R.) Copenhagen report NBI-HE-73-14 (1973)

[20] FIALKOWSKI (K.) and MIETTINEN (H.), Phys, Letters 43B, 493 (1973)

THOMAS (G.H.), Argonne report ANL/HEP 7251

[21] JENGO (R.), KRZYWICKI (A.), and PETERSSON (B.), paper 59

[22] DAO (F.T.), LACH (J.), and WHITMORE (J.), paper 283 ,

DAO (F.T.) and WHITMORE (J.), paper 284

[23] WEINGARTEN (D.), KOBA (Z.), Copenhagen reports (1973)

[24] BARSHAY (S.), Copenhagen reports 1973 BURAS (A.J.), DETHLEFSEN (J.M.) and KOBA (Z.) Copenhagen report NBI-HE-73-6
[25] LE BELLAC (M.), MEUNIER (J.L.) and PLAUT (G.) Nice report

[26] FRAZER (W.), PECCEI (R.), PINSKY (S.), and TAN (C.I), San Diego report USCD 1OP10-113

[27] WILSON (K.G.), Cornell University Preprint, CLNS-131 (1970)

FIALKOWSKI (K.), Phys, Letters 41B (1972) 379 FIALKOWSKI (K.) and MIETTINEN (H.), Phys. Letters 43B (1973) 61

HARARI ( $\overline{\text { H.) }}$ and RABINOVICI (E.), Phys, Letters 43B (1973) 49

QUTGG (C.) and JACKSON (J.D.), NAL-THY-93 LACH (J.;) and MALAMUD (E.), Phys, Letters $44 B$ (1973) 474

[28] VAN HOVE (L.), Phys. Letters 43B, (1973) 65

[29] KOBA (Z, ) and WEINGARTEN (D. ), CERN report ref. TH 1677 (1973)

[30] DAO (F.T.) et a1., Phys. Letters 45B (1973) 399 and 402

[31] FRAZER (W.) and SNIDER (D.), Phys. Letters (to be published ; HORN (D. ) MOSCHE (M.), NAL report $73 / 46 \mathrm{BHY}$ ROBERTS (R.G.) and ROY (D.), Rutherford report RL-73-065 T64

[32] BROMBERG (C.M.) et al., Rochester-Michigan report UMBC $72-14$ and VR 416 (1973); BARISH (S.J.) et al., ANL/HEP 7338 (1973)

[33] BERGER (E.L.), FOX (G.C.) and KRZYWICKI (A.), Phys. Letters 43B (1973) 132 . HANLON $\left(J_{.}\right)$et al., paper 151

[34] HORN (D.) and SCHWIMMER (A.), Nucl. Phys. B52, (1973) 627 DRIJARD (D.) and POKORSKI (s.), Phys. Letters 43B (1973) 509 .

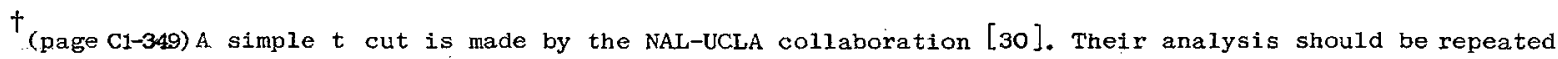
over a broad range of energies to see how the diffractive and non-diffractive subsamples change with $s$.
} 